\title{
El reciclaje de madera una herramienta para emprender y cuidar el planeta
}

\section{Wood recycling a tool to undertake and care for the planet}

Elías David Caisa Yucailla

Mario Patricio Padilla Martínez

Geovanny Fabricio Ríos Lara

Universidad Técnica de Ambato, Ecuador

Autor por correspondencia: eliasdcaisa@uta.edu.ec; mp.padilla@uta.edu.ec; geovannyfrios@uta.edu.ec

Fecha de recepción: 04 de julio del 2019 - Fecha de aceptación: 21 de noviembre del 2019

\section{Resumen}

Esta propuesta de gestión empresarial, se enfoca en la reutilización de un producto que normalmente es desechado luego de ser utilizado en la transportación de varios accesorios, especialmente de electrodomésticos, este desecho puede causar un impacto negativo en el medio ambiente ya que este material tiene un periodo de descomposición de muchos años. Las cifras preponderantes de este proyecto indican que existe mercado suficiente para explotar el reciclaje de madera de pino y convertir esta actividad en un modelo de negocio. El objetivo de este artículo es estudiar de qué manera el reciclaje de madera de pino (Palet), puede ser visto desde una perspectiva de negocio futuro. Se utilizó el método de investigación descriptivo porque permitió evaluar las características de la madera de pino y relacionarla como un potencial negocio para emprenderlo. Se aplicó una encuesta direccionada a los hogares para determinar su opinión de la reutilización de la madera de pino en productos novedosos, además de recurrir al criterio de diferentes profesionales que les gusta adquirir productos realizados con el pino, otro segmento de comercialización de estos productos realizados con el reciclaje puede ser, muebles y enseres de oficina, adornos entre otros. La investigación determinó resultados significativos sobre la necesidad de reciclar aportando las bases necesarias para crear negocios en el que ciertas familias puedan beneficiarse.

Palabras Clave: reciclaje; desechos; madera; negocio; ambiente

\begin{abstract}
This business management proposal, focuses on the reuse of a product that is normally discarded after being used in the transportation of various accessories, especially appliances, this waste can cause a negative impact on the environment since this material has a decomposition period of many years. The preponderant figures of this project indicate that there is sufficient market to exploit the recycling of pinewood and turn this activity into a business model. The objective of this article is to study how the recycling of pinewood (Pallet) can be seen from a future business perspective. The descriptive research method was used because it allowed to evaluate the characteristics of pine wood and relate it as a potential business to undertake it. A householddirected survey was applied to determine their opinion of the reuse of pinewood in novel
\end{abstract}


products, in addition to resorting to the criteria of different professionals who like to purchase products made with pine, another marketing segment of these products made with recycling can be, furniture and office supplies, ornaments among others. The investigation determined significant results on the need to recycle providing the necessary bases to create businesses in which certain families can benefit.

Key words: recycling; waste; wood; business; environment

\section{Introducción}

Fruto del consumismo por parte cada uno de los hogares y del sector productivo, se genera una gran cantidad de residuos sólidos que a la larga terminan en convertirse en basureros altamente contaminantes. Este artículo se fundamenta en los antecedentes sobre la disposición final de los residuos sólidos, por su limitada importancia provoca como resultado la contaminación ambiental y el riesgo al ser humano, provocada por sí mismo. (Allo \& Endara, 2015), pero con el estudio siguiente podemos determinar que los residuos se pueden reutilizar convirtiéndose como fuentes de ingreso a las familias al aparecer nuevos negocios que ayuden a contribuir a la matriz productiva y las familias.

El reciclaje no es un tema nuevo, se ha ido forjando a través del tiempo para crear conciencia con respecto a la sostenibilidad ambiental, siendo difícil crear y aplicar modelos macro ya que cada país desarrolla entornos diferentes limitando su efectividad. El deficiente apoyo económico, la falta de uso de tecnologías, incorrecta administración de un marco legal y el desinterés en una cultura responsable, son factores negativos que provocan un aumento de actividades no apropiadas para él ambiente. Las grandes industrias, tomados en cuenta como los principales agentes productores de residuos sólidos, que provoquen contaminación deben personificar un papel importante en la protección del medio ambiente, y aunque existen leyes que regulan y controlan la responsabilidad social de las empresas hacia el medio ambiente se puede palpar el deficiente compromiso por descartarlos responsablemente, es decir, investigar el método más adecuado para reciclar, reutilizar y reducir los residuos contaminantes, permitiendo la creación de nuevas fuentes económicas.

Se puede concretar que el problema que se ocasiona actualmente es la acumulación de palets de pinos obsoletos, derivados de actividades productivas que tienen las empresas importadoras y exportadoras, lo que ocasiona acumulación de residuos que pudiendo ser reutilizados no se les da ningún tratamiento. Este problema es causado por tres aspectos relevantes, el primero relacionado a una deficiente información que se le puede dar al uso de materiales reciclables, lo que ocasiona un incremento ambiental, la segunda causa es la carencia de prácticas de responsabilidad social, esto tiene efectos negativos ya que se produce desequilibrios en la sostenibilidad del ambiente y la economía de los países, la tercera causa se deriva de la deficiente innovación en actividades económicas que aporten al cambio de la matriz productiva, siendo su efecto la carencia de emprendimientos orientados a la elaboración de productos con materiales reciclados.

Se determina consecuentemente la pregunta de investigación es: ¿El reciclaje es una respuesta clave como un futuro negocio?

Para analizar la pregunta planteada, se detallan los objetivos: 
- Determinar un marco conceptual para definir la realidad del reciclaje

- Identificar los segmentos de mercado y cuáles son sus características

- Relacionar la iniciativa como un modelo de negocio reutilizando el reciclaje.

La materia prima utilizada para la fabricación de los pallets es la madera, debido a sus excelentes propiedades resistentes, reducido peso, economicidad y facilidad para reparar, reutilizar y reciclar, aspectos que convierten al pallet de madera en una herramienta de transporte y que fomente y facilite el desarrollo sostenible. Se puede afirmar esto, genéricamente, debido al empleo de un material de procedencia natural, renovable, sumidero de carbono atmosférico (aspecto que contribuye a mitigar las consecuencias perniciosas del efecto invernadero) y, particularmente, porque el sector del reciclado del pallet permite alargar las consecuencias positivas para la naturaleza que supone el secuestro y almacenamiento de carbono en la madera, destacando que un metro cúbico de este material alberga en torno a una tonelada de $\mathrm{CO} 2$ y es el único material constructivo que presenta un balance positivo al considerar este factor. (Solís, 2012)

Es importante esta investigación, ya que no existe una propuesta clara en materia de reciclaje en las empresas que importan o elaboran este tipo de producto y desarrollar responsabilidades para el ambiente y que ayuda a generar fuentes de ingresos, es factible porque existe la predisposición por parte de los empresarios, la comunidad (beneficiarios) implementando una cultura, técnicas y estrategias de reciclaje y con ello se logrará un impulso a la sociedad mejorando el medio ambiente mejorando el nivel y calidad de vida de la población. (Sanmartin, 2017)

Enfocados en la línea de investigación en el fortalecimiento de los actores de la económica y desarrollo empresarial, aplicando dentro del área del desarrollo económico y social, relacionado con la capacidad de los ingresos con emprendimientos nuevos para la creación de un nuevo producto (Moreno, 2018), con el reciclaje de los pallets se podrá elaborar productos tales como; jaulas para animales menores, casas para los canes, elaboración de útiles, muebles y enseres de oficina, para profesionales de las distintas áreas y de los consumidores que les guste la madera de pino; con ellos se quiere crear riqueza a fin de mantener la prosperidad o bienestar económico y social de sus habitantes, proponiendo modelos de negocio que ayuden a las familias del país.

\section{Fundamento Teórico}

\section{Modelo de Negocio}

(Osterwalder, 2018), manifiesta que; "Un modelo de negocio es la manera que una empresa o persona crea, entrega y captura valor para el cliente”. Gracias a Pizcar, los habitantes de muchas ciudades ya no necesitan un vehículo en propiedad, sino que pueden alquilar el coche que deseen por horas o días a cambio de una cuota de socio. Este modelo de negocio responde a las nuevas necesidades de los usuarios y a las inquietantes condiciones medioambientales.

\section{Tres elementos que debe tener un Modelo de Negocio:}


- Rentabilidad: Ningún negocio se monta para perder dinero, por tanto, debe generar utilidad

- Escalabilidad: Que hoy se pueda montar uno, mañana otro y así sucesivamente hasta tener un modelo que conquiste el mercado.

- Repetibilidad: Que se puedan estandarizar para poder hacerlos replicables en cualquier lugar, por ejemplo, los productos de franquicia.

De otra manera, cuando vaya a lanzar un producto o un servicio, estos deben también cumplir tres elementos que los debe hacer exitosos en el mercado, que son: Deseables, Viables $\boldsymbol{y}$ Rentables.

"Los clientes son el centro de cualquier modelo de negocio, ya que ninguna empresa puede sobrevivir durante mucho tiempo si no tiene clientes"

\section{Emprendimiento}

La característica del emprendedor es la de una persona autosuficiente que encuentra oportunidades, planifica y emprende con riesgo calculado sin perder su alegría, persistencia y sus dotes de liderazgo (Silva, De La Cruz, Lima, Cross, \& Rojas, 2016).

Sobre la actividad emprendedora dice Caniels citado por (Muzzio, 2017), que puede tener tres estados: 1) tener una idea prometedora; 2) socializar esa idea para lograr aceptación y recursos; 3 ) crearle a esa idea una innovación. La materialización de los emprendimientos, con sus riesgos y todo, se concreta en sentirse propietarios del negocio y tener la satisfacción de sentir que les pertenece y con ello sentirse productivos y en posesión de un bien de capital (Gonzalez, 2015).

En el proceso de identificar a los emprendedores, los investigadores coinciden en señalar que dentro de esa capacidad hay atributos como los de actitud, motivación y riesgo calculado, lo que hace de ellos personas especiales de entre la masa social (Cervilla \& Puente, 2013). También se suele decir que el emprender es un arte y como tal hay un espíritu de transformar lo que está deteriorado para aprovecharlo de la mejor manera; es maximizar la utilidad ilimitadamente y llegar a universalizarla (Fuller, 2013).

Emprender es innovar y allí tiene primerísima importancia el diseño, por eso quienes trabajan con la madera deben dar opciones estéticas y funcionales que satisfagan los requerimientos del cliente sin dejar de considerar el bajo impacto (Ramírez, 2018).

Por todas estas razones se considera la iniciativa empresarial como el eje principal que permite aglutinar otras virtudes creativas y que ayuda al avance innovador de la economía del mundo (Santos \& Brugni, 2014). Como vivimos la globalización en todas las actividades humanas, nuestro país no está al margen de esta actividad, por eso encontramos que en los estudios sobre emprendimientos (Padilla \& Quispe, 2017), que hizo "Global Entrepreneurship Monitor" ubicó a Ecuador como el segundo del mundo en este campo, con un $33.6 \%$ es decir, un emprendedor por cada tres personas (Pico, 2016). Con estos datos podemos evidenciar que el 
emprender no significa grandes empresas, sino aprovechar una necesidad para proponer una alternativa de trabajo.

\section{El reciclaje}

Los beneficios que tiene el reciclaje son muchos y se pueden enumerar desde el ambiental hasta el económico sin dejar de considerar el impacto positivo que tiene para la salud. El reciclaje protege el ambiente al no utilizar materia prima virgen y con ello se ayuda a proteger la salud y también el ahorro de energía (Largo, DeLongpre, \& Wight, 2013).

El reciclaje hace que el consumo de un material sea más lento y gracias a ello, existe la decisión de convertir esos materiales reciclados en sustentables, sin toxicidad y de larga vida útil (Putruele \& Veneziani, 2015). En las ciudades del mundo donde hay apoyo para el reciclaje encontramos en primera instancia dos grandes beneficiarios: los municipios y la industria (Hotta, Visvanathan, \& Kojima, 2016).

Nos dice Singer, citado por (Alcantara, Carneiro, Rodrígues, \& Costa Da Silva, 2015), que esta forma de producir a base del reciclaje es un proceso de economía solidaria para la producción con materias primas pequeñas.

Según Lumpkin y Dess, citado por (Do Reis, Muñoz, Correia, \& Pradella, 2013), la orientación de los emprendedores se da mediante tres aspectos que son: la voluntad de decidirse a asimilar riesgos; la capacidad innovadora y; la voluntad proactiva.

El reciclaje como parte de la sustentabilidad para mitigar la contaminación de sembríos, de ecosistemas y aumento de la pobreza, debe buscar procesos de innovación eficientes, cambios en las formas de consumo y frenar los efectos contaminantes, a la vez que, debe maximizar el reciclaje disminuyendo el crecimiento de utilización de nuevos materiales (Putruele \& Veneziani, 2015).

Entre los diversos beneficios que puede tener el reciclaje en los Estados Unidos, están el encontrar una solución al desecho generado y por otra parte darle nueva vida a un residuo sobre todo de los embalajes buscándoles una nueva utilidad (Klaiman, Ortega, \& Gamache, 2016).

\section{La materia prima, la madera}

La madera ha estado presente en la historia del hombre y ha ocupado un papel primordial para resolver sus necesidades como la vivienda, herramientas de trabajo, utensilios para sus alimentos, producir energía, calor y defensa. Modernamente es utilizada para fabricar papel, muebles, decoraciones, partes de aeronaves o como proveedor de sustancias para la industria química (De la Paz \& Dávalos, 2016).

La madera como recurso natural tiene un rol importante en la economía del mundo (González, y otros, 2015). Su gran utilidad como soporte estructural para construcciones hacen de la madera la mejor opción ya sea por su capacidad física, prestaciones dinámicas, oportunidad de diseño o creación de ambiente interior (Sotomayor, Suarez, \& Olguín, 2015). 
La madera tiene la capacidad de ofrecer al cliente soluciones a sus requerimientos con un producto que cubre las expectativas y puede ser hecho a la medida. Es por esto que el sector madera tiene futuro de crecimiento si los empresarios siguen las estrategias correctas para ser competitivos, contribuir al desarrollo y cumplir los parámetros medio ambientales para reducir la deforestación de los árboles naturales e incentivar la siembra de nuevos bosques (Salas \& Cortabarría, 2014).

Entre los materiales desechados por la sociedad, existen aquellos que en su composición intrínseca no han terminado su ciclo de vida y la madera es uno de ellos. En este tipo de reciclaje donde la innovación está presente se busca no sólo eliminar la basura, sino transformarla mediante el diseño en otro elemento que por sus características puede ofrecer prestaciones de utilidad y confort y de esta manera aportar a la sustentabilidad mediante la reutilización de materiales no perecibles (Holt \& Téllez, 2016).

En España ha existido el interés de clasificar las maderas por su nombre científico, aunque la realidad entre quienes se dedican a esta actividad es distinta ya que utilizan sus nombres comunes; existe un listado de los nombre comerciales según la reglamentación oficial, la UNE-EN 13556:2004 de maderas aconsejados en su uso (Villasante, Vignote, \& Ricardo, 2014)

El pino es la madera de uso común en la fabricación de pallets que sirven para el transporte internacional de mercaderías, los mismos que al llegar a sus destinos se convierten en desechos. La madera de pino es del tipo de las coníferas y existe gran variedad de ellas, dependiendo de la zona geográfica de producción se asocian nombres correspondientes que le han dado los comercializadores como es el caso de pino amarillo, para la procedente de la zona Norte de EEUU y la de pino tea, de la zona Sur (Villasante, Vignote, \& Ricardo, 2014).

\section{Compromiso social con el medio ambiente}

En una encuesta realizada en Santiago de Chile a jóvenes universitarios, se encontró que, en una abrumadora mayoría, tanto hombres como mujeres, señalan que los llamados a cuidar del medio ambiente son los gobiernos locales y nacional, empresarios y toda la ciudadanía (Pavez, León, \& Triadú, 2016). Todos los segmentos sociales deben ser partes interesadas e integrar los programas de reciclajes como gestores o técnicos en cada uno de sus campos, cada actor juega un papel importante y garantiza la sostenibilidad de los programas (Kian, Kohei, \& Mohd, 2015).

La participación de los actores comprometidos con el medio ambiente hace necesario que quienes tienen el poder político lideren los cambios y marquen el camino a seguir de los demás miembros sociales, y tal es el caso que los gobiernos de los Estados Unidos y Reino Unido que prohibieron mediante legislación el uso de micro plásticos en cosméticos y detergentes (Sutherland, Barnard, Broad, Connor, \& otros, 2017).

La humanidad afronta serios problemas con los residuos sólidos ya que su manejo se le está yendo de las manos y amenaza con comprometer la existencia de la vida misma. Según la "Agencia de Protección Ambiental de los Estados Unidos (USEPA)" citada por (Rolando, 
Carreño, Lucas, \& Cárdenas, 2016), los desechos no se gestionan correctamente y representan una seria amenaza para la salud humana ya que existe contaminación del agua y con ella se contamina todo el medio ambiente del planeta.

El mundo vive una crisis ambiental que proyecta consecuencias desastrosas si no se toman medidas a tiempo, en este contexto se hace necesario poner en práctica formas avanzadas de producir así como la utilización racional de elementos que no porque sean útiles para la humanidad se permita que desvirtúen la natural forma de convivir del hombre con su entorno, para ello está el modelo Biomímesis que se enfoca en la "ecología industrial y la economía ecológica" (Carvajal, 2017).

\section{El futuro de la sustentabilidad}

La historia del reciclaje es corta y como tal tiene mucho por delante en cuanto a desarrollarse como sistema y seguir aportando con beneficios que pueden ser incalculables, pero en la actualidad existen limitaciones que dependen del mayor o menor apoyo que brindan en cada país. El reciclaje como sistema para manejo de residuos sólidos encuentra limitaciones en ciudades del tercer mundo ya que otras están inmersas en programas integrales que comprenden la reducción, la reutilización, el reciclaje y la disposición final de desechos (Navarrete, 2016).

Dentro de las varias fortalezas que tiene la empresa del reciclaje se pueden resaltar que la generación de puestos de trabajo y la creación de una renta que la empresa privada tradicional no lo puede hacer, hacen de esta actividad un negocio que proyecta rentabilidad tanto en el presente como en el futuro (Arévalo \& Gomez, 2018). En Colombia el sector de madera y muebles que se desarrolla en las ciudades de Santa Marta, Barranquilla y Cartagena se encuentra constituido mayoritariamente como actividad personal o familiar y solamente unos pocos casos son de sociedades legalmente estructuradas. Hay empresas donde se realiza la actividad artesanalmente sin tener una estructura formal y una conformación legal (Salas \& Cortabarría, 2014).

El asesor especial del Secretario General de la ONU, Jeffrey Sachs, fundador del Proyecto de Desarrollo del Milenio, que busca el progreso económico de los países del tercer mundo con sustento ecológico de protección del medio ambiente, fundamentaba este proyecto en hacer que las naciones pobres reciban inversiones de las naciones del primer mundo en infraestructura para el desarrollo eco sustentable, y de esta forma hacer mucho más eficiente el progreso para la economía de la humanidad (Fuller, 2013).

La metodología utilizada sobre la valoración del ciclo de vida de los materiales utilizados en la producción industrial o de servicio, tiene un estándar para medir el impacto ambiental y son tres: la huella ecológica; la huella de agua y; la huella de carbono. Otras huellas como las de nitrógeno y la de emisiones, entre otras, no están estandarizadas (Elia \& Gnoni, 2016). Las operaciones de producción eco sostenibles necesitan tres fundamentos para operar en todo el proceso de trabajo, siendo de características integrales en el conjunto del proceso creativo y productivo, y se pueden definir como: lo económico, lo social y como tercero lo ambiental (Wu, Lv, Liang, \& Hu, 2016). 
Según Srtvastava, Fahey y Chistensen, citado por (Wu, Lv, Liang, \& Hu, 2016), el análisis de las teorías que estudian las oportunidades que brindan los recursos, resaltan que haya una ventaja en la competitividad por el hecho de la creación de un producto, en función de que se trata de una innovación que demuestra capacidad y habilidad. El reciclaje como actividad económica de crecimiento progresivo ha ido a la par de la necesidad de mano de obra preparada para este fin (Texeira, 2015). Para encontrar alternativas en cuanto a construcciones de estructuras con menor impacto ambiental, las investigaciones apuntar a la reutilización de materiales de desecho (Napolano, Menna, Asprone, Prota, \& Manfredi, 2015).

En el mundo se está imponiendo la tendencia de explorar modelos de negocios conocidos como de Economía Circular que es recuperar y revalorizar los residuos como materia prima para una nueva cadena de suministros (Elia \& Gnoni, 2016). La industria automotriz, así como la de embarcaciones desde sus inicios han utilizado la madera, ahora realizan investigaciones para aplicar el reciclaje de materiales compuestos, es decir, la mezcla de varios de ellos para crear otros específicos a sus necesidades (Page, 2016). Las afectaciones producidas al ecosistema con desequilibrios que repercuten en la vida diaria hacen necesario tomar acciones por parte de todos nosotros, comprometiéndonos cada uno en su respectiva área en favor del reciclaje (Putruele \& Veneziani, 2015). El compromiso ecológico debe ser llevado como un conjunto de acciones y no como ideas separadas que cada cual lleva por su lado, se trata de una acción científica donde aportan toda la humanidad con sus conocimientos específicos (Thakur, 2017).

\section{Metodología}

\section{Enfoque de la investigación:}

En la presente investigación se empleará la modalidad cuali-cuantitativa de acuerdo al paradigma critico- propositivo. Se determina cuantitativa porque confía la mediación numérica, el conteo y el empleo de la estadística descriptiva para el análisis de interpretación de resultados que permitirán verificar y solucionar la problemática. Además, es cualitativa porque se basa en métodos de recolección de datos sin medición numérica, es decir mediante la observación para reconstruir la realidad reciclaje de madera como herramienta para el emprendimiento.

\section{Modalidad Básica de la Investigación}

Se aplicaron las siguientes modalidades de investigación:

Investigación Bibliográfica o Documental: se realizó un análisis sobre la información escrita realizada en libros, revistas periódicos, artículos, otros.

Investigación de Campo: Se efectúa en el lugar y tiempo en que ocurren los fenómenos objetos de estudio, es decir en las empresas y en las familias interesadas en recurrir a una nueva fuente de ingresos económicos 


\section{Tipo de investigación}

Investigación Descriptiva: mediante esta investigación se pudo describir de una manera más clara como se manifiesta el reciclaje en el entorno y como se pude generar nuevos negocios que ayuden a mejorar las economías.

Los procedimientos utilizados en la investigación fueron en primer lugar la determinación de la muestra que se orientó a los hogares y a los empresarios, para ello se aplicó preguntas estructuradas en un cuestionario, en donde se identificó los siguientes bloques: 1) datos generales de los encuestados, edad, género, estado civil, posición laboral; 2) estructura de preguntas direccionadas a la comprensión del reciclaje de madera de pino; 3) relación de la necesidad de adquirir un producto reciclado. En segundo lugar, se estableció una serie de preguntas estructuradas para los propietarios de las empresas determinándose en orden de prioridad: 1) su conocimiento sobre la madera desechada; 2) la predisposición para la compra del producto; 3) el interés por su precio y la forma del producto. En tercer lugar, se determinó la muestra en el sector de comercio y manufactura, direccionados a los comerciantes productores de muebles, a los cuales se les aplicó un cuestionario estructurado cuyo contenido fue: 1) identificación de datos generales como ubicación del negocio y duración en esta actividad; 2) focalización con respecto a datos de sus ventas y gustos de sus clientes con respecto a sus productos más vendidos, 3) y una de las más importantes el interés de poner un negocio a base del reciclaje

El estudio señalado se efectuó en 5 meses, ya que se cubrió una zona geográfica no delimitada que estuvo orientada a identificar los negocios establecidos en la producción y comercialización de artículos de madera, además de identificar el segmento de mercado en la población agrupada en los hogares y en las veterinarias. Los aspectos señalados anteriormente permitieron relacionar las variables del estudio, aplicándose como herramienta estadística la tabulación en Excel, por considerar que los datos mientras más sencillos se encuentren mayor es la comprensión del tema, esta estadística descriptiva resultó significativa por los resultados encontrados.

\section{Resultados}

Los resultados más relevantes de esta investigación se detallan a continuación; la mayoría de la población se concentra en personas de 35 a 60 años de edad, lo que visualizó el pensamiento de la persona frente al reciclaje. 


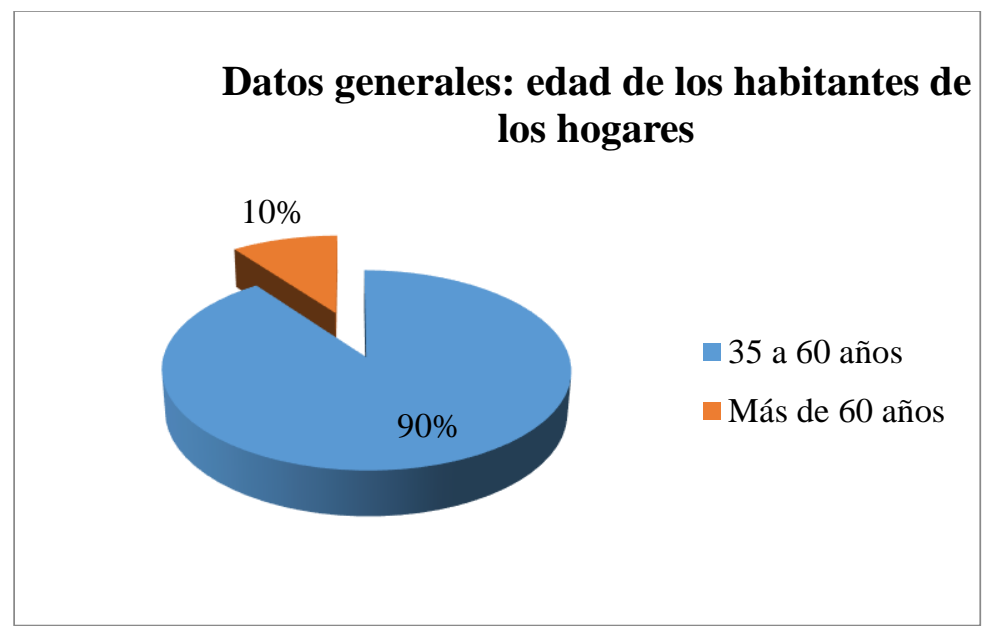

Gráfico \# 1 Se puede observar que la mayoría de los encuestados son personas que todavía esta dispuestas a ejecutar ciertas actividades que ayudan al crecimiento de la economía del país.

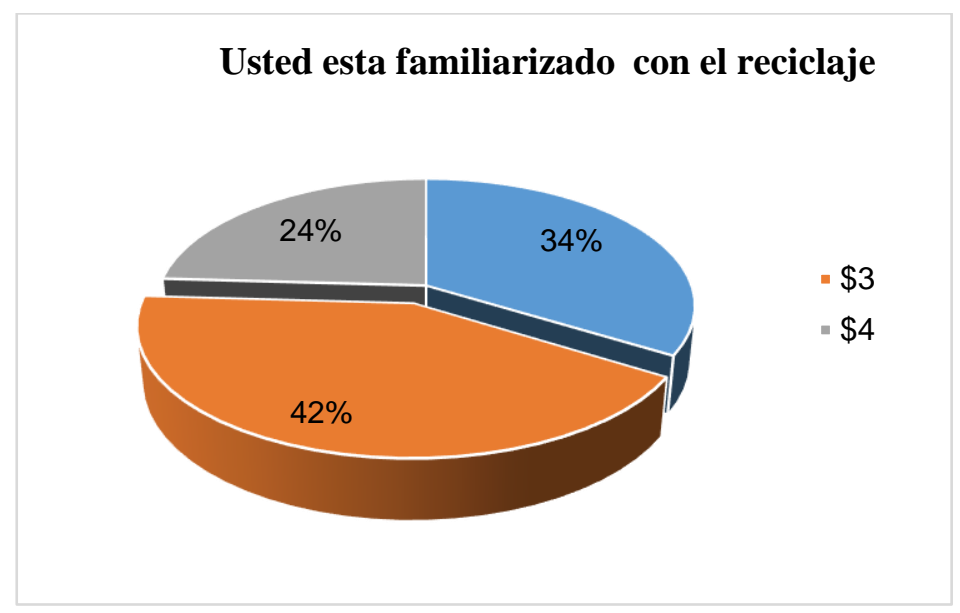

Gráfico \# 2 Se determinó en una mayoría que las personas si tienen conocimientos sobre el reciclaje, pero es el desconocimiento de cómo debo actuar frente a este beneficio por eso se pretende crear modelos de negocio.

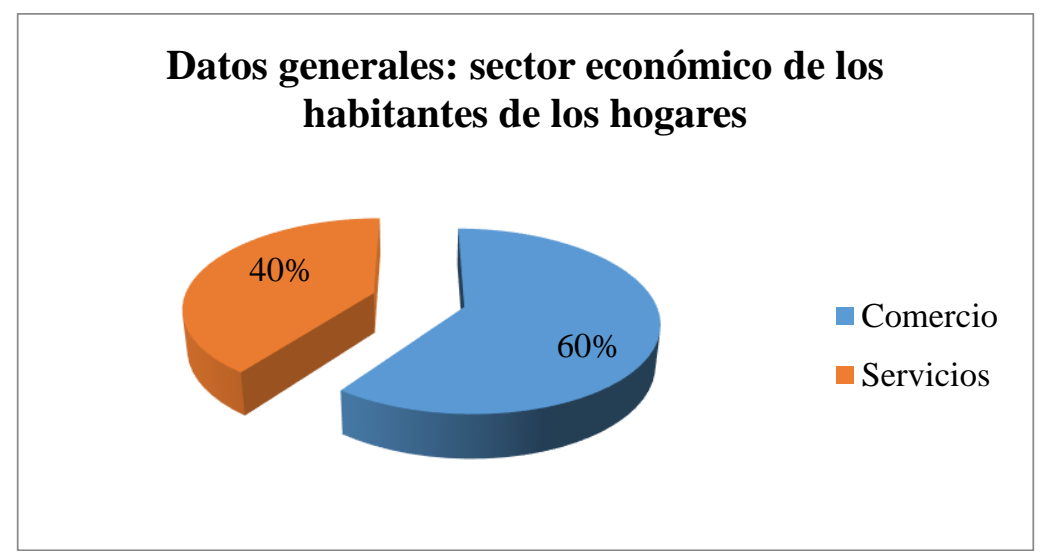

Gráfico \# 3 Se determinó que el sector económico en el que se encuentran trabajando las personas encuestadas es el comercio, este porcentaje es alto e indica la capacidad de pago de los posibles consumidores de los productos. 


\section{Uso del producto transformado en artículos de decoración para el hogar}

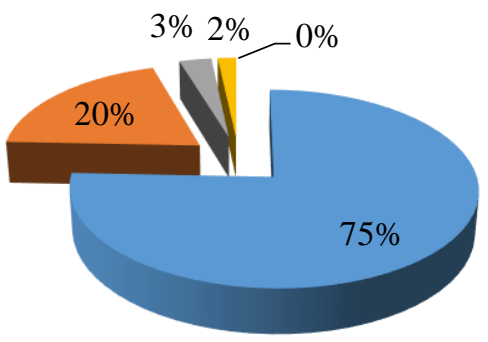

Totalmente de acuerdo

De acuerdo

Indiferente

Totalmente en desacuerdo

- Desacuerdo

Gráfico 4 Se detalló que la aceptación de la población frente a un producto reciclado es alta, pocas son las personas que señalan al reciclaje como una actividad productiva.

\section{Adquisición de algún artículo para el hogar elaborado con palets}

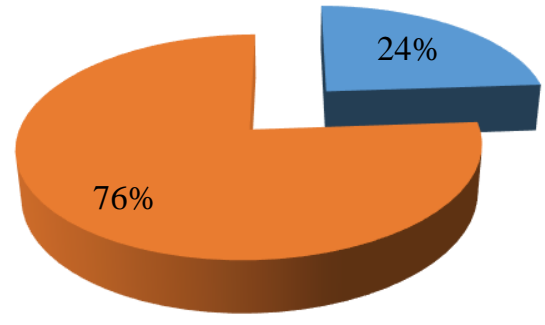

$\mathrm{Si}$

No

Gráfico 5 Se puede visualizar que la mayoría de los encuestados no poseían un producto reciclado en su hogar, esto indica la importancia del reciclaje en los hogares y el impacto que tiene aprovechar un recurso reutilizable.

\section{Usted formaria parte de los negocios que se pueden realizar con el reciclaje}
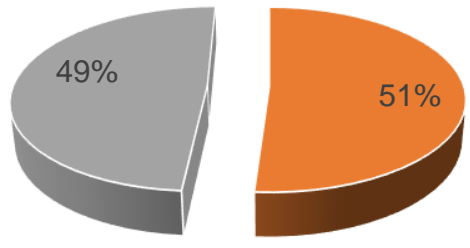

$=\mathrm{NO}$

Gráfico 6 Se identificó que las personas si están interesadas en ser parte de los nuevos negocios que se producen con el reciclaje ya que ayudan a fomentar las economías familiares 


\section{Compraría Ud., productos provenientes} del reciclaje por su:

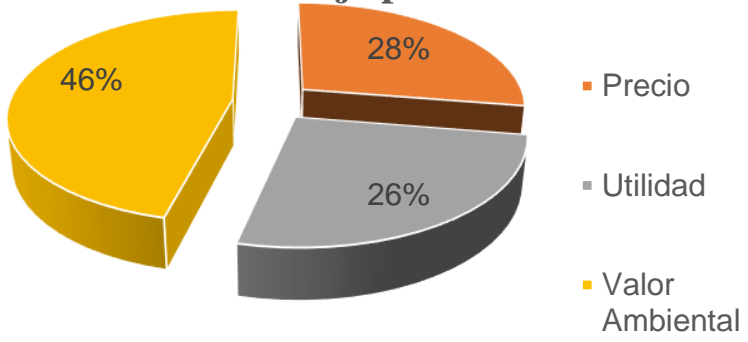

Gráfico 7 Se observa que los productos a base del reciclaje si tiene una aceptación por los usuarios ya que los usuarios están conscientes del daño ambiental y quieren corar estos productos como una estrategia de compensación al daño causado

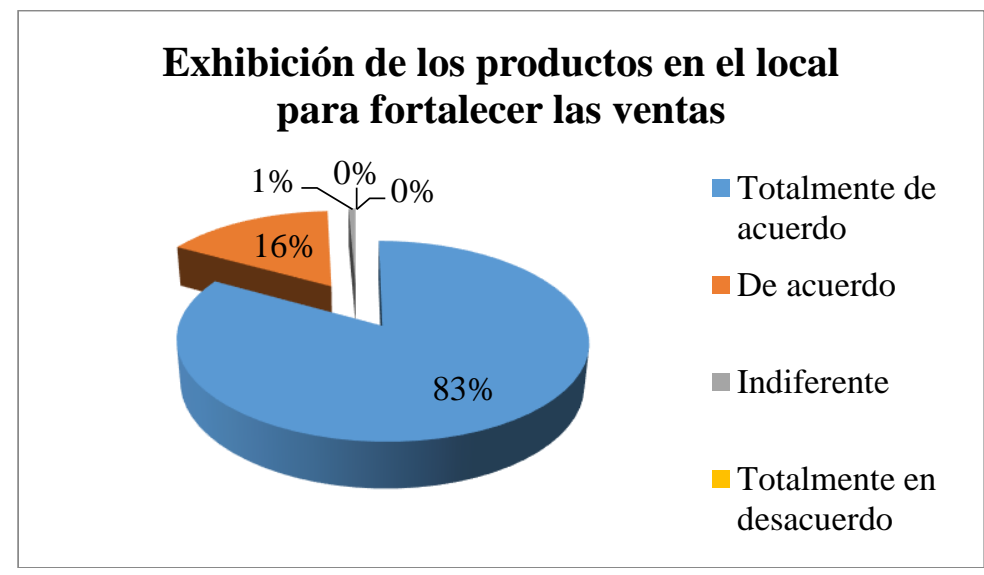

Gráfico 8 Se concreta que los comerciantes productores de muebles, necesariamente requieren de un sitio para exhibir sus productos, por lo que este sistema tradicional permite a los consumidores tener una visión más general del artículo, aspecto relevante para el empresario.

\section{Discusión}

Se detectó que la aplicación de las encuestas en diferentes segmentos, tienen semejanzas en su composición, así, por ejemplo, la edad de las personas abordadas es un referente para que el empresario futuro comience a visualizar cuál es el mercado adecuado para este tipo de producto reciclado. Actualmente se tiene deseo y necesidad de brindar mayor atención a las mascotas, especialmente del género femenino, aspecto relevante de la investigación, de igual forma, focalizar el sector económico al que pertenecen en su relación de dependencia o autonomía de negocio también da una pauta para comprender la composición del mercado potencial, el futuro empresario tiene entonces las herramientas adecuadas para iniciar sus operaciones.

Una cuestión fundamental es la aceptación de la población y la conciencia que tienen sobre el reciclaje, el obtener una idea de cómo desarrollar un artículo decorativo para los hogares es una idea aceptada, es importante destacar que solamente el 5\% de esta población encuestada es indiferente o está en desacuerdo con esta actividad. Al momento de recopilar información 
sobre la compra de algún artículo reciclado, la mayoría de la población no lo ha hecho, sin embargo, la conciencia de responsabilidad con el medio ambiente tiene un impacto mayor cuando se produce el deseo de compra y la percepción de que este producto permite la conservación de los árboles.

Con respecto a los empresarios establecidos en el sector servicios, se determina que los gustos y preferencias de sus clientes, le dan la pauta para aceptar esta idea novedosa, un alto porcentaje de personas propietarias del negocio de veterinarias establecen que los productos con madera de pino reciclados son novedosos y útiles para sus mascotas.

De otro lado, los propietarios de las empresas productoras y comercializadoras de muebles se establecen en su actividad de manera fuerte, por lo que su posicionamiento ya está concretado, sin embargo de aquello hay aún apertura para ingresar al mercado con un producto innovador, el empresario debe iniciar y asumir el riesgo en esta actividad, más aún si se tiene un mercado de proveedores constante, pues son los importadores y exportadores quienes derivan este material que puede ser reutilizado.

Finalmente, hay necesidad de destacar que el marketing a través del merchandising o de la publicidad, logra cimentar en la mente del consumidor ciertas marcas o productos, esto se demuestra en la investigación, porque al visitar los comercios de muebles se ocupan grandes espacios de exhibición, con ello se impulsa la creatividad de los propietarios, que aún deben mantener el interés de un mercado aún por explotar, más aún si la iniciativa es obtener un producto reciclado con innovación.

\section{Conclusiones}

Se determina que los objetivos de la investigación se cumplen, puesto que el reciclaje de madera de hoy en día, es un hábito muy importante ya que ayudaríamos a conservar el medio ambiente, la disminución de tala de árboles, siendo que la deforestación es uno de los principales causantes del calentamiento global, un daño crucial para la naturaleza y para todo aquel ser vivo. El reciclaje nos ofrece mil maneras de reutilizar lo que ya hemos cortado y usado (Mission, 2012), una estrategia de acrecimiento es la reutilización de los residuos en especial los de madera en el que se pueden generar pequeñas empresas que se dediquen a reutilizar estos residuos contribuyendo al crecimiento económico del país.

La madera en pino es una de las materias primas, que se puede moldear fácilmente, es duradero y se asemeja más a la naturaleza sin darles mayor proceso de transformación, aportando a un ambiente confortable, cómodo y con estilo retro. Cualquier decoración realizada con madera de pino, resulta económico y de calidad sin requerir mucho esfuerzo para renovar su aspecto y útil para cualquier espacio al que se desee acoplar. Los pallets de pino descartados por las empresas importadoras y exportadoras, son la materia prima adecuada para ser reutilizados para elaborar muebles para interior o exterior.

La recolección de desechos, sea desde el hogar o de las grandes industrias que producen cualquier cantidad de residuos sólidos es el inicio a la apertura por el cuidado ambiental. El reusar desechos de madera es una de las opciones se puede dar un nuevo acabado, darle una 
forma, originalidad, restáuralos y decorarlos. La transformación para este tipo de material se puede realizar con el objetivo de ofrecer un producto auténtico, renovado y útil para su hogar o para el espacio que esté elaborado.

Se puede aprovechar estos residuos de madera con la finalidad de generar nuevas empresas que den apertura a las familias a que puedan generar ingresos a sus hogares, tomando encuentra que el porcentaje de desempleo cada día más va aumentando en nuestro país, esta sería una solución para generar empleo y contribuir a la patria con un granito de área.

\section{Bibliografía}

Alcantara, B., Carneiro, G., Rodrígues, C., \& Costa Da Silva, M. (2015). Comprometimento organizacional e gestao de mens meteriais e patrimoniais em um empreedimento economico solidario: um estudo em uma cooperativa de reciclagem. RAM. REV. ADV. Mackenzie, 15-42.

Allo, S. M., \& Endara, O. Á. (2015). El Reciclaje De Basura Y El Desarrollo De Responsabilidades Para El Cuidado Ambiental. Ambato: Universidad Técnica de Ambato.

Arévalo, T. J., \& Gomez, G. A. (2018). Plan De Negocios Para Una Empresa De Reciclaje Que Vincule A Recicladores De Oficio Con Inversionistas Privados En La . Bogotá: PONTIFICIA UNIVERSIDAD JAVERIANA.

Carvajal, A. (2017). Tecnologías para el desarrollo sostenible. Revista de Filosofía de la Universidad de Costa Rica, 89-101.

Cervilla, M., \& Puente, R. (2013). Modelos de negocio de emprendimeintos por y para la base de la pirámide. Revista de Ciencias Sociales, 289-308.

De la Paz, C., \& Dávalos, R. (2016). Anatomía de la madera de seis especies de Pinus (Pinaceae) del estado de Durango-México. Madera y Bosques, 113-132.

Do Reis, J., Muñoz, P., Correia, C., \& Pradella, W. (2013). O papel da orientacao empreendedora no relacionamento entre rientacao para o mercado e desempenho empresarial: evidencias das pequenas empresas do comercio. REAd Revista Eletrónica de Administrazao, 115-138.

Elia, V., \& Gnoni, M. T. (2016). Measuring circular economy strategies through index methods: A critical analysis. Journal of Cleaner Production, 1-11.

Fuller, S. (2013). Never let a good crisis go to waste: moral entrepreneurship, or the fine art of recycling evil into good. Business Ethics: A European Review, 118-129.

Gonzalez, L. (2015). Constitución del sujeto como empresario de si: modos de subjetivación en el neoliberalismo. Nómadas 42, 197-212.

González, R., Rosales, M., Rocha, N., Gallegos, J., Moreno, M., \& Karchesy, J. (2015). Wood preservation using natural products. Madera y Bosques, 63-76.

Holt, J., \& Téllez, M. (2016). Aplicación de mobiliario diseñado a partir de materiales de desecho en propuestas de diseño de interiores. Jóvenes en la Ciencia, 1775-1779.

Hotta, Y., Visvanathan, C., \& Kojima, M. (2016). Recycling rate and target setting: challenges for standardized measurement. Mater Cycles Waste, 14-21.

Kian, T., Kohei, W., \& Mohd, T. (2015). Assessment of the sustainability level of community waste recycling program in Malaysia. Master Cycles Waste, 598-605.

Klaiman, K., Ortega, D., \& Gamache, C. (2016). Consumer Preferences and Demand for Packaging Material and Recyclability. Agricultural \& Applied Economics, 1-25. 
Largo, W. E., DeLongpre, D., \& Wight, J. (2013). The Efficacy of a Theory-Based, Participatory Recycling Intervention on a College Campus. Journal of Enviromental Health, 26-31.

Mission, C. (4 de Octubre de 2012). Como puede reciclarse la madera? Obtenido de www.ehowenespanol.com $>$ Hobbies

Moreno, C. L. (2018). Creación de una empresa dedicada a la elaboración de muebles con palets de pino reciclados en el Cantón Ambato. Ambato: UTA.

Muzzio, H. (2017). Individuo, Lideranza e Cultura: Evidencias de uma Gestao da Criatividade. RAC, Río de Janeiro, 107-124.

Napolano, L., Menna, C., Asprone, D., Prota, A., \& Manfredi, G. (2015). Life cycle environmental impact of different replaaacement options for a typical old flat roof. Life Cycle Sustainability Asseement, 694-708.

Navarrete, P. (2016). De cartoneros a recicladores urbanos. El rol de las políticas locales en mejorar la sustentabilidad de los recolectores de base. Investigaciones Regionales, 83106.

Osterwalder, A. (13 de febrero de 2018). Open Mind. Obtenido de https://www.bbvaopenmind.com/economia/empresa/que-es-un-modelo-de-negocio-ycomo-hacerlo-efectivo/: https://www.bbvaopenmind.com/economia/empresa/que-es-unmodelo-de-negocio-y-como-hacerlo-efectivo/

Padilla, M. M., \& Quispe, O. A. (2017). Modelo de Gestión de Emprendimdeintio Universitario. Matanzas: Universidad de Matanzas.

Page, T. (2016). Composite materials and their recycling. i-managers Journal on Material Science, 37-51.

Pavez, I., León, C., \& Triadú, V. (2016). Jóvenes universitarios y medio ambiente en Chile: Percepciones y comportamientos. Revista Latinoamericana de Ciencias Sociales, 14351449.

Pico, L. (2016). El emprendimiento por necesidad, una ventana hacia el desarrollo de oportunidades de negocios. Innova, 142-147.

Putruele, J., \& Veneziani, M. (2015). Sustentabilidad, diseño y reciclaje. Cuaderno 53, 123-131.

Ramírez, M. J. (2018). la importancia de la creatividad e innovación al emprender. Ecuador: Crece Mujer.

Rolando, L., Carreño, A., Lucas, R., \& Cárdenas, F. (2016). Aporte a la Gestiíon Integral de Residuos Sólidos no Peligrosos en la ESPAM-MFL. Espam Ciencia, 159-165.

Salas, K., \& Cortabarría, L. (2014). Análisis competitivo del sector madera y muebles de la región Caribe de Colombia. Prospect, 79-89.

Sanmartin, R. G. (2017). El reciclaje: un nicho de innovación y emprendimiento con enfoque ambientalista. Revista Universidad y Sociedad, 8.

Santos, A., \& Brugni, T. (2014). Empreendedorismo na perspectiva das Empresas de fomento ás Pequenas Empresas. Turismo y gestión de Estudios, 19-25.

Silva, D. S., De La Cruz, P., Lima, N., Cross, N., \& Rojas, A. (2016). Entrepreneurial Potential and Success in Business: a Study on Elements of Convergence and Explanation. RAM Rev Adm Mackenzie, 188-215.

Solís, T. J. (2012). El reciclado de palets: una solución integral para los residuos. Quito: Interempresas.

Sotomayor, J., Suarez, G., \& Olguín, J. (2015). Efecto del tratamiento higro-térmico en las características acústicas de la madera de Quercus acytophylla Liebm. Madera y Bosques, 139-156. 
Sutherland, W., Barnard, P., Broad, M., Connor, B., \& otros, y. (2017). A 2017 Horizon Scan of Emerging Issues for Global Conservation and Biological Diversity. Trends in Ecology \& Evolution, 31-40.

Texeira, K. (2015). Trabalho e perspectivas na percepcao dos catadores de materiais reciclaveis. Psicologtía \& Sociedade, 98-105.

Thakur, M. (2017). Putting Community Ecology in a Better Order. Trends in Ecology \& Evolution, 6-7.

Villasante, A., Vignote, S., \& Ricardo, B. (2014). Análisis estadistico de los nombres comerciales de maderas en España. Madera y Bosques, 59-70.

Wu, H., Lv, K., Liang, L., \& Hu, H. (2016). Mearuring perfomance of sustainable manufacturing with recyclable wastes: A case from China's iron and steel industry. Omega, 1-10. 